\title{
Defective (interfering) viral genomes re-explored: impact on antiviral immunity and virus persistence
}

\author{
Tomaz B Manzoni \& Carolina B López ${ }^{*, 1}$ \\ ${ }^{1}$ Department of Pathobiology, School of Veterinary Medicine, University of Pennsylvania, Philadelphia, PA 19104, USA \\ *Author for correspondence: lopezca@upenn.edu
}

Defective viral genomes (DVGs) are natural products of virus replication that occur in many positive and negative sense RNA viruses, including Ebola, dengue and respiratory syncytial virus. DVGs, which have severe genomic truncations and require a helper virus to replicate, have three well-described functions: interference with standard virus replication, immunostimulation, and establishment of virus persistence. These functions of DVGs were first described almost 50 years ago, yet only recent studies have shown the molecular intersection between their immunostimulatory and pro-persistence activities. Here, we review more than half a century of scientific literature on the immunostimulatory and pro-persistence functions of DVGs. We highlight recent advances in the field and the critical role DVGs have in both the acute and long-term virus-host interactions.

First draft submitted: 6 February 2018; Accepted for publication: 3 May 2018; Published online: 12 June 2018

Keywords: defective viral genomes $\bullet$ immunostimulation $\bullet$ interference $\bullet$ RNA virus $\bullet$ virus persistence

\section{DVGs, a historical perspective}

Defective viral genomes (DVGs) were first described in the early 1940s by Preben Von Magnus when he observed a decrease in infectivity of influenza virus passaged at high titers [1]. Von Magnus posited that particles of 'incomplete' virus were produced when viruses were expanded at high titers, and that these particles interfered with viral replication. His hypothesis was supported by the identification in virus stocks of a component with a lower sedimentation rate that associated with interference. This component was named 'Von Magnus particles' [2]. Follow-up experiments demonstrated that the production of Von Magnus particles was independent of the number of infective particles used in the infection [3]. Instead, it depended on the ratio of infectious $\left(\mathrm{ID}_{50}\right)$ to noninfectious particles in the original stock [4]. In the late 1950s, incomplete and interfering particles of Rift Valley fever virus were discovered in viruses passaged in mice at high concentrations [5] and during the 1960s, they were described in several other RNA viruses, including vesicular stomatitis virus (VSV), lymphocytic choriomeningitis virus, and Sendai virus (SeV) [6-11]. However, it was not until the late 1960s that a distinct RNA species shorter than the standard viral genome was reported in incomplete interfering viruses. The amount of this smaller genomic RNA correlated with the ratio of infectious to noninfectious particles [12-14]. It was then speculated that the Von Magnus particles resulted from errors during viral genome replication or during its incorporation into the virion $[13,15]$. Incomplete viral RNA genomes contained in interfering particles would later be known as defective interfering (DI) particles and, more recently, DVGs [16].

In 1970, Alice Huang and David Baltimore coined the term defective interfering particles, or DIPs, and defined DI particles as follows: "they contain normal viral structural proteins; they contain a part of the viral genome; they can reproduce in the presence of helper virus; they interfere specifically with the intracellular replication of nondefective homologous virus" [17]. Based on infections with VSV and influenza virus, Huang and Baltimore introduced the theory of DI 'waves' to explain the asynchronous cycles of DI particles and standard virus observed in persistently infected cultures (details of this theory in the following sections). Importantly, Huang and Baltimore proposed that DI particles played a critical role in determining the course of natural viral infections, including the establishment of virus persistence [17]. 
During the 1970s and 80s DI particles were described in many other RNA viruses, including poliovirus, rabies, measles, human parainfluenza virus 3, Semliki Forest virus and Sindbis virus [18-27]. It was quickly evident that the presence and amount of DI particles correlated with the establishment of persistently infected cells in vitro [9,19,28-34] and in vivo [35-38]. This was followed by the description of increased survival of infected mice in infections containing DI particles $[5,39,40]$ and by observations that DI particles enhanced the production of interferon (IFN) during infections $[26,30,41-43]$. By the 1990s, it was clear that most, if not all, RNA viruses could produce DI particles when grown at high multiplicity of infection, including the important human pathogens Ebola, respiratory syncytial virus (RSV) and mumps [44-46].

Despite strong data supporting important biological roles for DI particles, the relevance of these viral products to natural infections was questioned since their discovery. Moreover, DI particles were considered artifacts of in vitro infections and thus irrelevant to natural infections. Authors frequently discussed that despite the interesting properties of DI particles, they are not naturally produced in vivo and are likely caused by highly artificial methods of passaging the virus $[5,47]$. This line of thought, together with the lack of appropriate technology to identify and distinguish DI particles from the standard virus, largely limited research on DI particles to their use as tools for studying virus replication and as potential antivirals. Recent renewed interest in studying the role of DI particles during natural viral infections and viral persistence was largely motivated by the identification of DVGs in clinical samples [48,49], demonstrating that they indeed occur during natural infections.

\section{Interference \& immunostimulation by DVGs}

Multiple theories for how DVGs interfere with the replication of standard virus have been tested, including competition for viral receptors, competition for viral components needed for replication, and the induction of IFN [11,30,41,50,51]. These theories are founded on basic knowledge of the structure and properties of DVGs. Though the factors leading to some viruses producing more DVGs than others remain unknown, DVGs form when the viral polymerase loses processivity falling off the template genome and re-attaching elsewhere to complete replication [16]. This alteration during replication leads to truncations of the nascent viral genome resulting in the production of short replication defective genomes. Truncated viral genomes appear in two primary forms: deletion and copyback. Deletion DVGs are formed when the polymerase detaches from the template strand and re-attaches downstream, resulting in the production of DVGs that share their $3^{\prime}$ and $5^{\prime}$ ends with the full-length viral genome. Copyback DVGs are formed when the polymerase detaches from the template and reattaches to the nascent strand, creating a complementary end to the $5^{\prime}$ end of the viral genome. The shorter length of DVGs combined with promoters with increased affinity for viral polymerase in copyback DVGs favor the theory that interference is achieved via competition for viral components, including the viral polymerase (Figure 1A) [11,51-53].

It is well documented that DVGs of several viruses are strong inducers of IFN and they are considered the primary stimuli of antiviral immunity in many infections (Figure 1B) [26,48,54-59]. DVGs activate the intracellular RIG-I-like receptors and turn on the expression of IFNs and proinflammatory cytokines such as IL-1 $\alpha$, TNF, and IL-6. In addition, DVG stimulation optimizes the antigen presentation capacity of specialized antigen presenting cells that initiate adaptive immunity $[16,48,57,58,60]$. Moreover, accumulating evidence indicates that the immunostimulatory activity of DVGs is maintained in vivo and during natural infections. In mice infected with the respiratory viruses $\mathrm{SeV}$, influenza, or RSV, IFN and proinflammatory cytokines are strongly induced only after DVGs have accumulated to detectable levels $[48,56]$. Detection of DVGs in respiratory secretions of children infected with RSV correlates with expression of antiviral genes [48], and highly pathogenic influenza virus isolates that fail to induce potent antiviral responses in humans have an impaired ability to generate DVGs [49].

Because of their immunostimulatory activity DVGs have been tested as antivirals and vaccine adjuvants [61,62]. As antivirals, DVGs are expected to interfere with standard virus replication, thereby delaying virus spread and allowing the host time to mount a response [61]. This concept has been extensively proven in mice during infection with Semliki Forest virus, VSV, and influenza virus [39,63,64]. In addition, vaccines complemented with DVGs delivered subcutaneously, intramuscularly or intranasally show improved antibody production and increased protection from virus challenge $[65,66]$. Moreover, DVGs are present in live attenuated vaccines against polio, measles and influenza viruses [67-70] and are thought to impact their protective activity. Recent work from our laboratory identified a specific secondary structure on SeV DVGs that mediates their strong immunostimulatory activity [71]. Oligonucleotides containing this unique immunostimulatory motif can stimulate protective immune responses during vaccination [66] further illustrating the potential of harnessing DVGs as strong immunostimulants in vivo. 


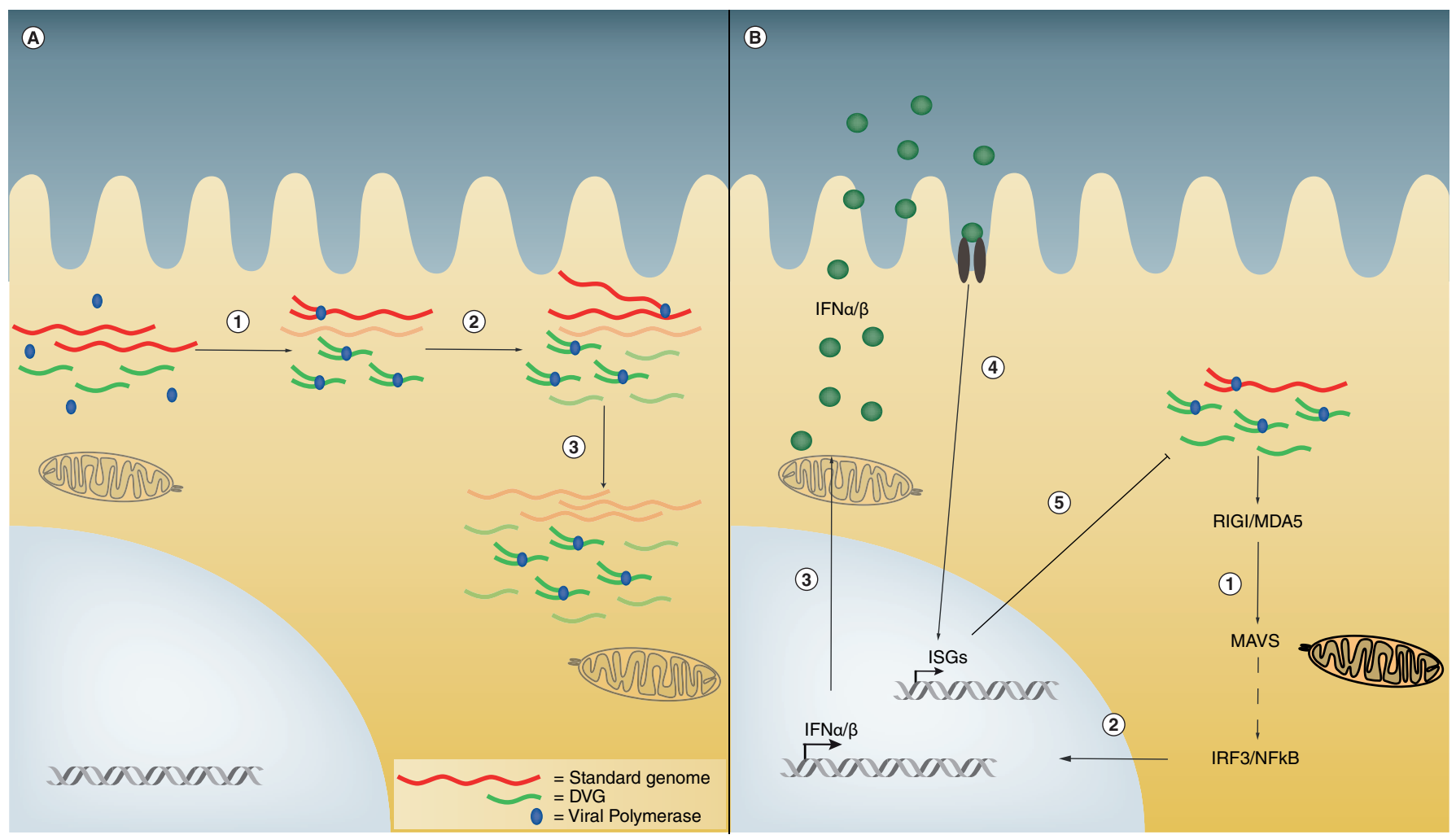

Figure 1. Defective viral genome interference by competition for viral components and interferon. (A) Competition for viral products occurs in cells that contain several copies of standard virus and DVGs. A limited available amount of polymerase and associated proteins randomly binds to viral genomes to begin the replication process (1). Data indicate that viral promoters on DVGs bind the viral polymerase with higher affinity than promoters in standard virus. As DVGs are shorter than standard virus they are also synthesized more rapidly and thus quickly accumulate (2). With a combination of faster transcription and stronger affinity for polymerase DVGs eventually outcompete standard virus to become the predominant species (3), thereby interfering with standard virus replication. (B) In interference by IFN, infected cells first detect DVGs through the RNA sensors RIG-I or MDA5 (1). Signaling through the adaptor protein MAVS leads to IRF3 and NFKB activation and translocation to the nucleus (2). These molecules stimulate the production and secretion of IFN $\alpha / \beta$ (3) that act in an autocrine or paracrine manner (4) to produce ISGs that inhibit viral replication (5).

DVG: Defective viral genome; IFN: Interferon; ISG: Interferon stimulated gene; MAVS: Mitochondria antiviral-signaling protein.

DVG immunostimulation is also an important factor in modulation of infections in insects. Similar to the pattern recognition receptors RIG-I and MDA5 in mammals, insects have Dicer-2, which senses viral RNA and processes it to produce siRNAs that confer antiviral immunity in insects [72]. Dicer-2 acts preferentially on DVGs relative to standard genome and lead to the control of viral replication and longer survival of infected mosquitos. These observations indicate that the immunostimulatory activity of DVGs is widespread and may have a significant impact in the spread of arboviruses such as chikungunya and Sindbis.

The impact of the immunostimulatory and interfering activities of DVGs for virus spread and clearance during natural infections remains unknown. One possibility is that DVGs act to limit the extent of the infection, thereby delaying the onset of debilitating disease symptoms that would prevent individuals from coming into contact with other susceptible hosts, or in extreme cases kill the host. The strong evidence for DVGs promoting virus persistence argues that keeping the host healthy is not the sole mechanism for viruses to benefit from DVGs. New data provide clues as to how immune stimulation and persistence, two seemingly opposite phenomena, occur in response to DVGs.

\section{The role of DVGs in persistence}

A growing number of RNA viruses, previously thought to be acutely infecting, are described to persist in humans, including RSV, human metapneumovirus, Zika, chikungunya, and Ebola [44,73-79]. The mechanisms leading to the establishment and maintenance of persistence of these viruses is poorly understood and contrast with other persistently infecting viruses. For example, retroviruses, such as HIV, integrate within their host's genome, and 
DNA viruses, such as herpes, establish episomes in dividing host cells nuclei and produce latent proteins that maintain the persistent infection [80]. RNA viruses are instead though to remain in the hostile environment of the cytoplasm in the absence of latent viral proteins that help maintain their persistent status. In addition, it is unclear whether persistent RNA viruses produce infectious viral particles either continuously or in response to stimulus as observed in other persistent viruses such as Epstein-Barr virus or HIV [80].

RNA virus persistence is usually pathogenic and associates with the development and exacerbation of chronic diseases. Perhaps the most well studied persistent RNA virus is measles. In persistent measles infections, patients develop subacute sclerosing panencephalitis, a neurologic condition that is often fatal [81]. Other viruses have been associated with chronic diseases, although a causal effect has not been demonstrated. Human metapneumovirus and RSV have been implicated in both asthma and chronic obstructive pulmonary disease [82], and chikungunya virus is associated with chronic arthralgia [78]. In some cases, persistent viruses remain transmissible [36,47,78,81,83,84]. Various RNA viruses persist in immuneprivileged sites including the brain, eyes and testes, and some are capable of being sexually transmitted long after recovery from the disease [76,79,85]. Persistently infected individuals may act as viral reservoirs allowing tourists, emergency healthcare workers, and other travelers to inadvertently carry and spread deadly viruses. For example, there is accumulating data on reactivation and spread of persistent Ebola virus [83,85]. Epidemiological data from the 2014 Ebola outbreak show that a recovered patient transmitted virus 482 days post onset of symptoms infecting 13 different individuals [83]. These epidemiological and biological data highlight the public health hazard of persistent highly pathogenic RNA viral infections.

Although it is well established that DI particles containing DVGs lead to persistently infected cell cultures $[9,19,28-$ 34,37], limited data support a role for DVGs in the establishment of persistent infections in vivo. One study reported that mice co-inoculated with Semliki Forest virus and DI particles established a persistent infection through an unknown mechanism [37]. Another study identified measles DVGs in the brain of humans that died due to subacute sclerosing panencephalitis [81]. DVGs may directly lead to persistence, be required for maintenance of persistence, or be produced but not affect persistence. Until recently, the only model of the impact of DI particles on persistence was suggested by Alice Huang in 1970 based on the cycling between high production of standard virus and DI particles during infections in vitro [17]. According to this theory, DI particles arise slowly during virus replication until they reach high concentrations and become predominant. These dominant DI particles interfere with standard virus replication and actively reduce the amount of standard virus. In this process, some cells are infected by standard virus and reinitiate the cycle. Other cells are infected only by DI particles, thus yielding no virus. During the next cycle of infection, DI particles are again produced slowly and accumulate repeating the waving pattern of virus production. Asynchronous cycling of DI particles and standard virus occurs in many persistent infections in vitro [86-88] and in vivo [5,88]. The cycling of DI particles and standard virus occur in a predictable pattern and has been mathematically modeled using variations of the predator-prey model $[89,90]$. Interestingly, in some persistent infections the amount of DI particles appears constant [91]. What drives these cyclic patterns in some viruses but not others, and whether host factors such as the infected cell-type influences the cycling pattern remain unknown.

A molecular mechanism for the establishment of persistence by DVGs was recently reported by our laboratory [92]. Using RNA in situ fluorescent hybridization (RNA FISH) we discovered heterogeneity in the content of viral genome species in epithelial cells infected with SeV in vitro. Some cells are enriched in DVGs, others in standard viral genomes. Cells with a high content of DVGs engage the mitochondria antiviral-signaling protein to induce the production of IFNs and other proinflammatory molecules, including TNF (Figure 2). TNF has two wellcharacterized opposing functions during infection depending on the signaling pathway it engages. If it signals through TNFR1, it acts as a proinflammatory and proapoptotic factor [93]. In contrast, if it signals through TNFR2, it acts as a pro-survival factor [93]. DVG-high cells express TNFR2 and many of its downstream signaling molecules, including the TNF receptor associated factor 1 (TRAF1). Autocrine TNF thereby extends the survival of DVG-high cells and the infection can persist for months [92]. In contrast, cells enriched in standard viral genome express TNFR1 and TNF promotes their death. A similar phenotype was observed in infections with RSV [92]. This mechanism explains the paradoxical stimulation of both antiviral immunity and establishment of persistence by DVGs and reveals a previously overlooked strategy for host-virus co-existence.

Though DVGs lead to persistent infections, evidence for other mechanisms of RNA virus persistence exists. For example, during parainfluenza virus infection high expression of neuraminidase in infected cells leads to the depletion of viral attachment factors preventing cell to cell fusion and cell death, thereby promoting persistence independently of DVGs [94]. Thus, it is unlikely that DVGs are the only mechanism for the establishment of RNA virus persistence. Host factors, such as cell and tissue types or immunological status of the patient also have a large 


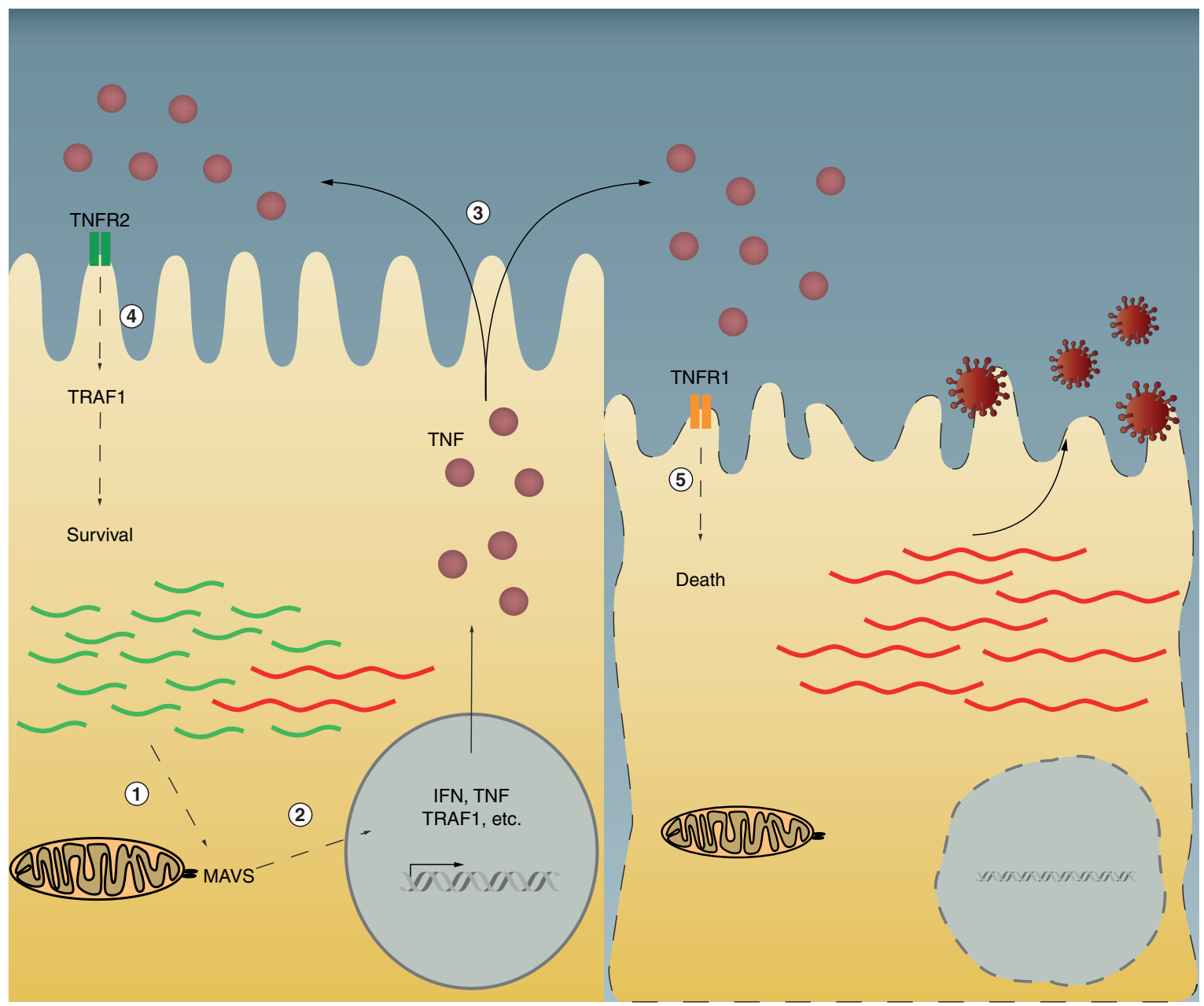

Figure 2. Mechanism for the establishment of persistent infections through defective viral genome

immunostimulation. Defective viral genome (DVGs) are sensed by the RIG-I-like receptors RIG-I and MDA5 and activate the downstream signaling component MAVS (1). MAVS signaling leads to the production of TNFR2, TRAF1, IFN, and TNF (2). IFN and TNF are secreted to act in an autocrine or paracrine manner (3). In DVG-high cells expressing TNFR2 and TRAF1, a prosurvival pathway is activated and virus persistence is established. In cells high in standard virus genome but with few or no DVGs the TNFR1 pathway is activated leading to cell death (4).

IFN: Interferon; MAVS: Mitochondria antiviral-signaling protein.

impact on the establishment of persistent infections. How host and viral factors, specifically DVGs, interact is the subject of active investigation.

It remains unclear how the enhanced survival of DVG-high cells leading to persistence fits into the DVG and standard virus cycling observed in many infections. Two nonmutually exclusive models may be envisioned: cycling may occur at the intracellular level, where each infected cell goes through cycles of standard viral genome or DVG enrichment driven by competition and interference with the viral replication machinery (Figure 3A). In this model, a single cell infected by a standard virus accumulates DVGs that will eventually take over the replication machinery thereby reducing standard virus replication to the verge of elimination. With limited viral polymerase available and failure to produce more due to interference, production of DVGs ceases allowing the standard virus to resurge and take over. This model makes the assumptions that cells enriched in standard virus can transition to a DVG-high status before they are killed, and that DVGs are eliminated once polymerase levels are reduced; cycling may occur at the population level, where individual infected cells will determine the composition of the pool of standard virus or DI particles available for infection of new cells (Figure 3B). In this model, cells infected with a standard virus may either die or transition to a DVG-high content. Cells transitioning into a DVG-high state shed both standard virus and DVGs until reaching a high DVG status where viral polymerase complexes are no longer available and 
(A)

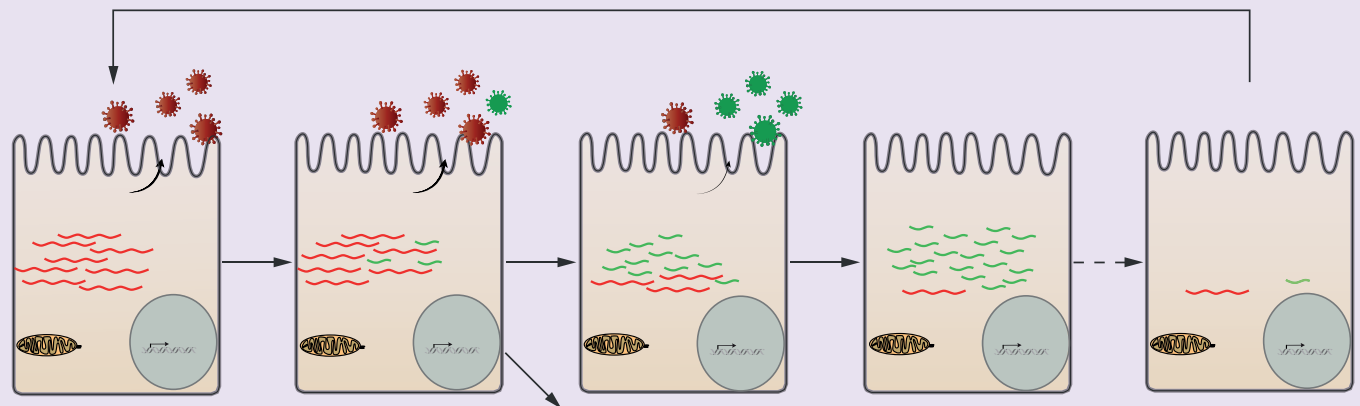

(B)
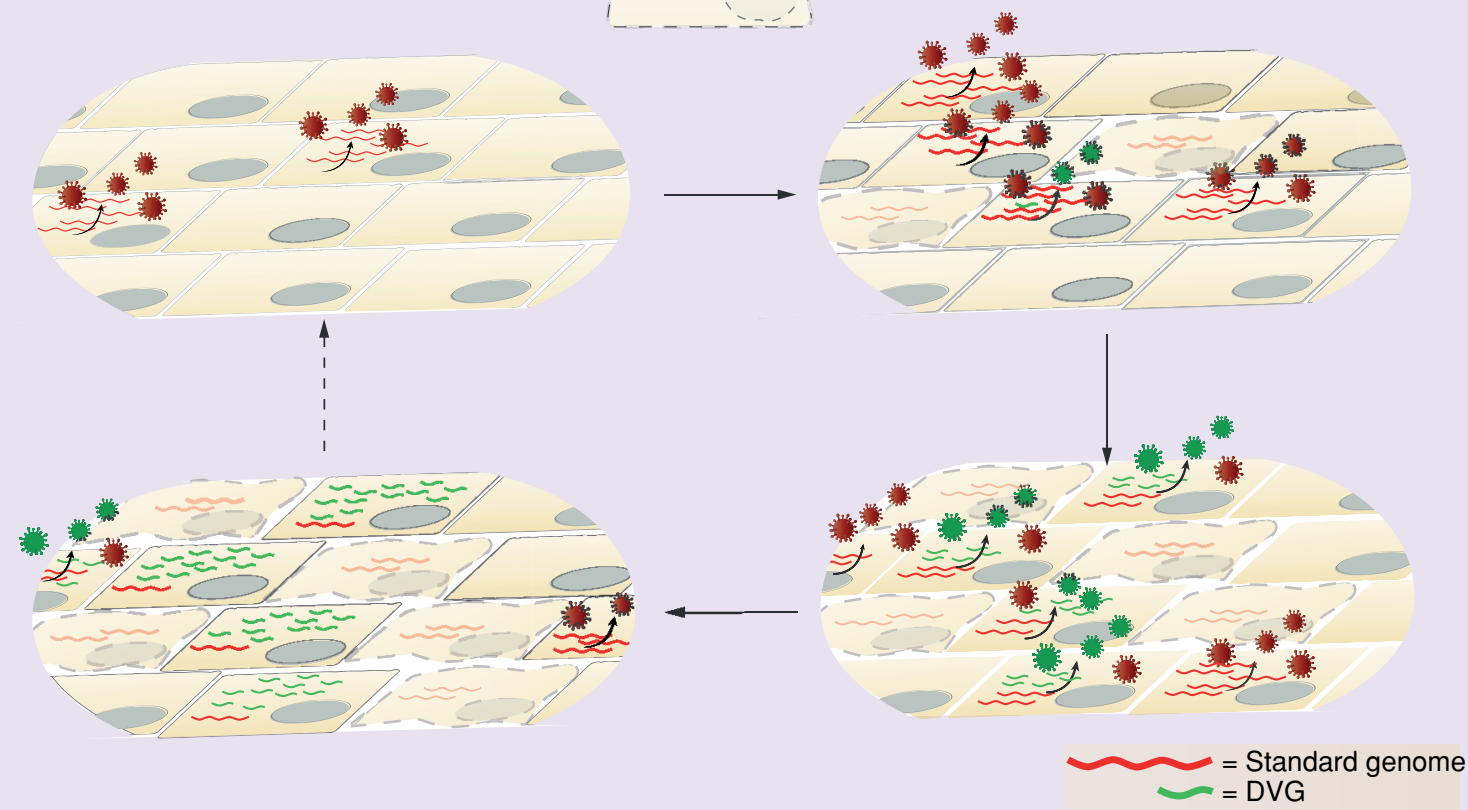

Figure 3. Models for defective viral genome cycling. (A) Intracellular cycling begins with a cell infected with standard virus. Upon virus replication DVGs slowly accumulate and are shed in Defective interfering (DI) particles. Cells that accumulate DVGs may escape cell death and instead transition to a DVG-high status where virus is no longer produced and viral proteins are not longer made due to interference. As a result, viral RNA decreases and the cycle is reinitiated given that DVGs are somehow eliminated. (B) In a population model, cycling also begins with a standard virus infection. As the infection propagates, DVGs form and DI particles are shed. Cells failing to produce DVGs die while cells able to replicate DVGs survive accumulating DVGs until reaching a high content accompanied by low standard virus. As DVGs interfere with viral protein production within cells, DVG and virus production diminishes. Cells that have died from infection with standard virus are eventually replaced by new cells allowing the cycle to begin again as standard virus infects those new uninfected cells. DVG: Defective viral genome.

no more virus is produced. As most standard virus-high cells die and DVG-high cells begin shedding less virus, dead cells are replaced allowing for infection to take hold. As these new cells are naive, standard virus must infect them in order to establish a productive infection and reinitiate the cycle. This model does not specifically consider other forms of virus spread, such as cell-to-cell fusion, cell division, or virus transfer through cytoplasmic channels, although similar dynamics are expected to apply in these conditions. 


\section{Conclusion}

Despite decades of work on DVGs and their functions, much remains unknown. As there are different types of DVGs, it is conceivable that immunostimulation, interference and persistence exist as separate functions for at least some. In addition, in light of recent developments, it is important to consider how new models of DVG-standard virus dynamics align with older models of DVG-induced persistence. It is possible that persistently infected cells are not stable but behave dynamically and switch between DVG-high and low states (Figure 3B). This switch would be dependent on the degradation of viral RNAs yet it is unclear how this would occur and whether DVGs are degraded at different rates than standard viral genome. Data for this dynamic intracellular behavior remain limited yet DVG cycling as described in the literature refers to population dynamics as opposed to cellular dynamics. It is thus possible that population cycling is independent of cellular cycling as long as persistently infected cells continue producing viruses. Further work must be done to elucidate cellular dynamics from population dynamics.

\section{Future perspective}

Recent data on DVG biology and dynamics during infection raise a number of new questions. Out of particular interest is whether all RNA viruses can persist. $\mathrm{SeV}$ is a widely used model virus for the Mononegavirales order of viruses, which includes paramyxoviruses, such as parainfluenza virus, pneumoviruses, such as RSV, filoviruses, such as Ebola and rhabdoviruses, such as VSV. Interfering DVGs have been characterized for all these viruses and many have been observed to persist $[7,22,44,45,73,91]$. Whether the same mechanism for establishment of persistence applies to similar viruses of this order requires further research and the relevance of this mechanism during natural infections needs to be determined. In addition, many positive sense RNA viruses have been shown to produce DVGs and persist; yet it remains unclear whether they use a similar strategy for persistence. With evidence of DVGs functioning as immunostimulators in insects, it is also important to consider how arboviral DVGs impact infections of mammals versus insects and what is their role during virus spread and transmission between species.

Many additional questions on the contributions of DVGs to antiviral immunity and persistence remain unanswered: what controls DVG generation? What regulates their heterogeneous distribution in an infected population? Do surviving cells remain DVG-high or is there a mechanism for switching to a virus-producing cell? Do persistent viruses adopt unique localizations in order to escape the cell's antiviral sensors? How does DVG production impact viral protein production and what are the impacts on infection? What are the determinants for DVGs stimulating the TNFR2 prosurvival pathway as opposed to the TNFR1 pathway? Though a clear correlation between DVGs and persistence exists, this remains an understudied field. The ability for RNA viruses to persist raises public health concerns and additional questions about the biology of persistence: how long does a persistently infected individual shed virus? Is the individual constantly shedding virus or do events such as stress and subsequent homologous or heterologous infections lead to reactivation of virus? What are the cellular reservoirs? With renewed interest in the study of DVGs, we hope to see many of these questions answered in the near future. In addition, identifying factors that influence establishment and maintenance of persistence is critical to manage the onset and impact of persistent infections. For example, it is important to know if persistent virus may reactivate during immunosuppression or whether some patients are more susceptible to viral persistence, thus being at a higher risk to develop chronic pathologies. As defective virus detection methods become more sensitive and more persistent RNA viruses are identified, we will be better equipped to understand how DVGs contribute to the persistence of important human pathogens.

\section{Acknowledgements}

We thank all Lopez Lab members that read and commented on this article.

\section{Financial \& competing interests disclosure}

Work in the Lopez Lab related to this article was funded by NIH grants Al127832 and Al083284. The authors have no other relevant affiliations or financial involvement with any organization or entity with a financial interest in or financial conflict with the subject matter or materials discussed in the manuscript apart from those disclosed.

No writing assistance was utilized in the production of this manuscript.

\section{Open access}

This work is licensed under the Attribution-NonCommercial-NoDerivatives 4.0 Unported License. To view a copy of this license, visit http://creativecommons.org/licenses/by-nc-nd/4.0/ 
Executive summary

Historical studies on defective viral genomes

- Discovery of defective viral genomes (DVGs) more than half a century ago originated from the observation of decreased infectivity from virus passaged at high titers.

- DVGs were shown to occur in most RNA viruses.

- Critical functions of DVGs have been described including correlation with persistent infections, interferon induction and decreased severity of infection in mice.

- Lack of evidence for DVGs occurring in natural infections delayed progress in the field until a recent resurgence of interest accompanied by better technologies to distinguish standard and defective viral genomes.

Interference \& immunostimulation

- DVGs can interfere with full length virus replication by competing for the viral polymerase.

- For many viruses DVGs have been described as the primary stimulus for induction of antiviral immune responses. This observation highlights the potential for DVGs to be used as vaccine adjuvants or antivirals.

- Immunostimulatory functions of DVGs are ubiquitous with data suggesting similar function in model mammals, insects and humans.

DVGs \& persistence

- Unlike other viruses known to persist, RNA viruses do not form episomes or integrate into host genome.

- RNA virus persistence associates with many chronic diseases and may lead to re-emergence of viruses from persistent reservoirs.

- In vivo data of persistence are scarce yet data do support DVGs drivers of persistent infections in animal models.

- Asynchronous cycling between high production of DVGs and full length virus is often observed during persistence.

- A mechanism for the establishment of persistence by DVGs has recently been proposed. In this model, DVG enriched cells trigger a prosurvival response to TNF while cells high in standard full length virus follow the TNF apoptotic pathway. It remains unclear how ubiquitous this mechanism is and what other mechanisms may exist for the establishment of persistence.

\section{References}

Papers of special note have been highlighted as: $\bullet$ of interest; $\bullet \bullet$ of considerable interest

1. Magnus PV. Studies on interference in experimental influenza. Arkiv För Kemi, Mineralogi, Och Geologi 24, 1-6 (1947).

2. Gard S, Von Magnus P, Svedmyr A, Birch-Andersen A. Studies on the sedimentation of influenza virus. Arch. Gesamte Virusforsch. 4(5), 591-611 (1952).

3. Cairns HJ, Edney M, Fazekas De St Groth S. Quantitative aspects of influenza virus multiplication. J. Immunol. 69(2), 155-181 (1952).

4. Beale AJ, Finter NB. The infectivity of chorio-allantoic membrane influenza virus and incomplete influenza virus by the six-hour soluble antigen production test. J. Hyg. (Lond.) 54(1), 68-78 (1956).

5. Mims CA. Rift Valley Fever virus in mice. IV. Incomplete virus; its production and properties. Br. J. Exp. Pathol. 37(2), 129-143 (1956).

6. Bellett AJ, Cooper PD. Some properties of the transmissible interfering component of vesicular stomatitis virus preparations. J. Gen. Microbiol. 21, 498-509 (1959).

7. Cooper PD, Bellett AJ. A transmissible interfering component of vesicular stomatitis virus preparations. J. Gen. Microbiol. 21, 485-497 (1959).

8. Sokol F, Neurath AR, Vilcek J. Formation of incomplete sendai virus in embryonated eggs. Acta Virol. 8, 59-67 (1964).

9. Lehmann-Grube F, Slenczka W, Tees R. A persistent and inapparent infection of L cells with the virus of lymphocytic choriomeningitis. J. Gen. Virol. 5(1), 63-81 (1969).

10. Huang AS, Greenawalt JW, Wagner RR. Defective T particles of vesicular stomatitis virus. I. Preparation, morphology, and some biologic properties. Virology 30(2), 161-172 (1966).

11. Huang AS, Wagner RR. Defective T particles of vesicular stomatitis virus. II. Biologic role in homologous interference. Virology 30 (2), 173-181 (1966).

12. Duesberg PH. The RNA of influenza virus. Proc. Natl Acad. Sci. USA 59(3), 930-937 (1968).

13. Pons M, Hirst GK. The single- and double-stranded RNA's and the proteins of incomplete influenza virus. Virology 38(1), 4 (1969).

14. Kingsbury DW, Portner A, Darlington RW. Properties of incomplete Sendai virions and subgenomic viral RNAs. Virology 42(4), 857-871 (1970).

15. Kingsbury DW, Portner A. On the genesis of incomplete Sendai virions. Virology 42(4), 872-879 (1970).

16. Lopez CB. Defective viral genomes: critical danger signals of viral infections. J. Virol. 88(16), 8720-8723 (2014). 
17. Huang AS, Baltimore D. Defective viral particles and viral disease processes. Nature 226(5243), 325-327 (1970).

-• This seminal paper defines defective interfering particles and speculate on their significance and impact on pathogenesis.

18. Cole CN, Smoler D, Wimmer E, Baltimore D. Defective interfering particles of poliovirus. I. Isolation and physical properties. J. Virol. 7(4), 478-485 (1971).

19. Kawai A, Matsumoto S, Tanabe K. Characterization of rabies viruses recovered from persistently infected BHK cells. Virology 67(2), 520-533 (1975).

20. Wiktor TJ, Dietzschold B, Leamnson RN, Koprowski H. Induction and biological properties of defective interfering particles of rabies virus. J. Virol. 21(2), 626-635 (1977).

21. Hall WW, Martin SJ, Gould E. Defective interfering particles produced during the replication of measles virus. Med. Microbiol. Immunol. 160(2-3), 155-164 (1974).

22. Murphy DG, Dimock K, Kang CY. Defective interfering particles of human parainfluenza virus 3. Virology 158(2), 439-443 (1987).

23. Bruton CJ, Kennedy SI. Defective-interfering particles of Semliki Forest Virus: structural differences between standard virus and defective-interfering particles. J. Gen. Virol. 31(3), 383-395 (1976).

24. Bruton CJ, Porter A, Kennedy SI. Defective-interfering particles of Semliki Forest virus: intracellular events during interference. J. Gen. Virol. 31(3), 397-416 (1976).

25. Fuller FJ, Marcus PI. Interferon induction by viruses. Sindbis virus: defective-interfering particles temperature-sensitive for interferon induction. J. Gen. Virol. 48(Pt 2), 391-394 (1980).

26. Fuller FJ, Marcus PI. Interferon induction by viruses. IV. Sindbis virus: early passage defective-interfering particles induce interferon. $J$. Gen. Virol. 48(1), 63-73 (1980).

27. Kennedy SI, Bruton CJ, Weiss B, Schlesinger S. Defective interfering passages of Sindbis virus: nature of the defective virion RNA. J. Virol. 19(3), 1034-1043 (1976).

28. Roux L, Waldvogel FA. Establishment of Sendai virus persistent infection: biochemical analysis of the early phase of a standard plus defective interfering virus infection of BHK cells. Virology 112(2), 400-410 (1981).

29. Roux L, Holland JJ. Role of defective interfering particles of Sendai virus in persistent infections. Virology 93(1), 91-103 (1979).

30. Sekellick MJ, Marcus PI. Persistent infection. I interferon-inducing defective-interfering particles as mediators of cell sparing: possible role in persistent infection by vesicular stomatitis virus. Virology 85(1), 175-186 (1978).

- Describes interferon inducing capabilities of defective viral genomes (DVGs) and correlation with establishment of persistence.

31. Schmaljohn C, Blair CD. Persistent infection of cultured mammalian cells by Japanese encephalitis virus. J. Virol. 24(2), 580-589 (1977).

32. De BK, Nayak DP. Defective interfering influenza viruses and host cells: establishment and maintenance of persistent influenza virus infection in MDBK and HeLa cells. J. Virol. 36(3), 847-859 (1980).

33. Andzhaparidze OG, Bogomolova NN, Boriskin YS, Bektemirova MS, Drynov ID. Comparative study of rabies virus persistence in human and hamster cell lines. J. Virol. 37(1), 1-6 (1981).

34. Kennedy JC, Macdonald RD. Persistent infection with infectious pancreatic necrosis virus mediated by defective-interfering (DI) virus particles in a cell line showing strong interference but little DI replication. J. Gen. Virol. 58(Pt 2), 361-371 (1982).

35. Barrett AD, Cross AJ, Crow TJ, Johnson JA, Guest AR, Dimmock NJ. Subclinical infections in mice resulting from the modulation of a lethal dose of Semliki Forest virus with defective interfering viruses: neurochemical abnormalities in the central nervous system. J. Gen. Virol. 67(Pt 8), 1727-1732 (1986).

36. Baczko K, Liebert UG, Billeter M, Cattaneo R, Budka H, Ter Meulen V. Expression of defective measles virus genes in brain tissues of patients with subacute sclerosing panencephalitis. J. Virol. 59(2), 472-478 (1986).

37. Atkinson T, Barrett AD, Mackenzie A, Dimmock NJ. Persistence of virulent Semliki Forest virus in mouse brain following co-inoculation with defective interfering particles. J. Gen. Virol. 67(Pt 6), 1189-1194 (1986).

38. Popescu M, Lohler J, Lehmann-Grube F. Infectious lymphocytes in mice persistently infected with lymphocytic choriomeningitis virus. Z Naturforsch C 32(11-12), 1026-1028 (1977).

39. Rabinowitz SG, Huprikar J. The influence of defective-interfering particles of the PR-8 strain of influenza A virus on the pathogenesis of pulmonary infection in mice. J. Infect. Dis. 140(3), 305-315 (1979).

40. Barrett AD, Dimmock NJ. Modulation of Semliki Forest virus-induced infection of mice by defective-interfering virus. J. Infect. Dis. 150(1), 98-104 (1984).

41. Marcus PI, Sekellick MJ. Defective interfering particles with covalently linked [+/-]RNA induce interferon. Nature 266(5605), 815-819 (1977).

42. Paucker K, Cantell K, Henle W. Quantitative studies on viral interference in suspended L cells. III. Effect of interfering viruses and interferon on the growth rate of cells. Virology 17, 324-334 (1962).

43. Johnston MD. The characteristics required for a Sendai virus preparation to induce high levels of interferon in human lymphoblastoid cells. J. Gen. Virol. 56(Pt 1), 175-184 (1981). 
44. Calain P, Monroe MC, Nichol ST. Ebola virus defective interfering particles and persistent infection. Virology 262(1), 114-128 (1999).

45. Treuhaft MW, Beem MO. Defective interfering particles of respiratory syncytial virus. Infect. Immun. 37(2), 439-444 (1982).

46. Andzhaparidze OG, Bogomolova NN, Boriskin Yu S, Drynov ID. Chronic non-cytopathic infection of human continuous cell lines with mumps virus. Acta Virol. 27(4), 318-328 (1983).

47. Viola MV, Scott C, Duffy PD. Persistent measles virus infection in vitro and in man. Arthritis Rheum. 21(5 Suppl.), S47-S51 (1978)

48. Sun Y, Jain D, Koziol-White CJ et al. Immunostimulatory defective viral genomes from respiratory Syncytial virus promote a strong innate antiviral response during infection in mice and humans. PLoS Pathog. 11(9), e1005122 (2015).

49. Vasilijevic J, Zamarreno N, Oliveros JC et al. Reduced accumulation of defective viral genomes contributes to severe outcome in influenza virus infected patients. PLoS Pathog. 13(10), e1006650 (2017).

- Indicates correlation between DVGs and disease outcome.

50. Portner A, Kingsbury DW. Homologous interference by incomplete Sendai virus particles: changes in virus-specific ribonucleic acid synthesis. J. Virol. 8(4), 388-394 (1971).

51. Salinas Y, Roux L. Replication and packaging properties of short Paramyxovirus defective RNAs. Virus Res. 109(2), 125-132 (2005).

52. Stampfer M, Baltimore D, Huang AS. Ribonucleic acid synthesis of vesicular stomatitis virus. I. Species of ribonucleic acid found in Chinese hamster ovary cells infected with plaque-forming and defective particles. J. Virol. 4(2), 154-161 (1969).

53. Calain P, Roux L. Functional characterisation of the genomic and antigenomic promoters of Sendai virus. Virology 212(1), 163-173 (1995).

54. Dimmock NJ, Kennedy SI. Prevention of death in Semliki Forest virus-infected mice by administration of defective-interfering Semliki Forest virus. J. Gen. Virol. 39(2), 231-242 (1978).

55. Shingai M, Ebihara T, Begum NA et al. Differential type I IFN-inducing abilities of wild-type versus vaccine strains of measles virus. J. Immunol. 179(9), 6123-6133 (2007).

56. Tapia K, Kim WK, Sun Y et al. Defective viral genomes arising in vivo provide critical danger signals for the triggering of lung antiviral immunity. PLoS Pathog. 9(10), e1003703 (2013).

57. Yount JS, Kraus TA, Horvath CM, Moran TM, Lopez CB. A novel role for viral-defective interfering particles in enhancing dendritic cell maturation. J. Immunol. 177(7), 4503-4513 (2006).

58. Strahle L, Garcin D, Kolakofsky D. Sendai virus defective-interfering genomes and the activation of interferon-beta. Virology 351(1), 101-111 (2006).

59. Ho TH, Kew C, Lui PY et al. PACT- and RIG-I-dependent activation of type I interferon production by a defective interfering RNA derived from Measles virus vaccine. J. Virol. 90(3), 1557-1568 (2015)

60. Yount JS, Gitlin L, Moran TM, Lopez CB. MDA5 participates in the detection of paramyxovirus infection and Is essential for the early activation of dendritic cells in response to sendai virus defective interfering particles. J. Immunol. 180(7), 4910-4918 (2008).

61. Dimmock NJ, Easton AJ. Defective interfering influenza virus RNAs: time to re-evaluate their clinical potential as broad-spectrum antivirals? J. Virol. 88(10), 5217-5227 (2014).

62. Dimmock NJ, Easton AJ. Cloned defective interfering Influenza RNA and a possible pan-specific treatment of respiratory virus diseases. Viruses 7(7), 3768-3788 (2015).

63. Barrett AD, Guest AR, Mackenzie A, Dimmock NJ. Protection of mice infected with a lethal dose of Semliki Forest virus by defective interfering virus: modulation of virus multiplication. J. Gen. Virol. 65(Pt 11), 1909-1920 (1984).

64. Holland JJ, Doyle M. Attempts to detect homologous autointerference in vivo with influenza virus and vesicular stomatitis virus. Infect. Immun. 7(4), 526-531 (1973).

65. Martinez-Gil L, Goff PH, Hai R, Garcia-Sastre A, Shaw ML, Palese P. A sendai virus-derived RNA agonist of RIG-I as a virus vaccine adjuvant. J. Virol. 87(3), 1290-1300 (2013).

66. Mercado-Lopez X, Cotter CR, Kim WK et al. Highly immunostimulatory RNA derived from a Sendai virus defective viral genome. Vaccine 31(48), 5713-5721 (2013).

67. Bellocq C, Mottet G, Roux L. Wide occurrence of measles virus subgenomic RNAs in attenuated live-virus vaccines. Biologicals 18(4), 337-343 (1990).

68. Mclaren LC, Holland JJ. Defective interfering particles from poliovirus vaccine and vaccine reference strains. Virology 60(2), 579-583 (1974).

69. Singanayagam A, Zambon M, Lalvani A, Barclay W. Can defective interfering RNAs affect the live attenuated influenza vaccine? Author' reply. Lancet Infect. Dis. 17(12), 1235-1236 (2017).

70. Xue J, Chambers BS, Hensley SE, Lopez CB. Propagation and characterization of influenza virus stocks that lack high levels of defective viral genomes and hemagglutinin mutations. Front. Microbiol. 7, 326 (2016).

71. Xu J, Mercado-Lopez X, Grier JT et al. Identification of a natural viral RNA motif that optimizes sensing of viral RNA by RIG-I. MBio 6(5), e01265-15 (2015) 
72. Poirier EZ, Goic B et al. Dicer-2-dependent generation of viral DNA from defective viral genomes modulats immune control of RNA virus infection in insects. Cell Host Microbe 23(3), 353.e8-365.e8 (2018).

73. Sikkel MB, Quint JK, Mallia P, Wedzicha JA, Johnston SL. Respiratory syncytial virus persistence in chronic obstructive pulmonary disease. Pediatr. Infect. Dis. J. 27(10 Suppl.), S63-S70 (2008).

74. Falsey AR, Formica MA, Hennessey PA, Criddle MM, Sullender WM, Walsh EE. Detection of respiratory syncytial virus in adults with chronic obstructive pulmonary disease. Am. J. Respir. Crit. Care Med. 173(6), 639-643 (2006).

75. Liu Y, Haas DL, Poore $S$ et al. Human metapneumovirus establishes persistent infection in the lungs of mice and is reactivated by glucocorticoid treatment. J. Virol. 83(13), 6837-6848 (2009).

76. Tan JJL, Balne PK, Leo YS, Tong L, Ng LFP, Agrawal R. Persistence of Zika virus in conjunctival fluid of convalescence patients. Sci. Rep. 7(1), 5 (2017).

77. Labadie $\mathrm{K}$, Larcher $\mathrm{T}$, Joubert $\mathrm{C}$ et al. Chikungunya disease in nonhuman primates involves long-term viral persistence in macrophages. J. Clin. Invest. 120(3), 894-906 (2010).

78. Borgherini G, Poubeau P, Jossaume A et al. Persistent arthralgia associated with chikungunya virus: a study of 88 adult patients on reunion island. Clin. Infect. Dis. 47(4), 469-475 (2008).

79. Chughtai AA, Barnes M, Macintyre CR. Persistence of Ebola virus in various body fluids during convalescence: evidence and implications for disease transmission and control. Epidemiol. Infect. 144(8), 8 (2016).

80. Thorley-Lawson DA. EBV persistence-introducing the virus. Curr. Top. Microbiol. Immunol. 390(Pt 1), 151-209 (2015).

81. Sidhu MS, Crowley J, Lowenthal A et al. Defective measles virus in human subacute sclerosing panencephalitis brain. Virology 202(2), 10 (1994).

82. Martin JG, Siddiqui S, Hassan M. Immune responses to viral infections: relevance for asthma. Paediatr. Respir. Rev. 7(Suppl. 1), S125-S127 (2006).

83. Diallo B, Sissoko D, Loman NJ et al. Resurgence of Ebola virus disease in Guinea linked to a survivor with virus persistence in seminal fluid for more than 500 days. Clin. Infect. Dis. 63(10), 1353-1356 (2016).

- Highlights public health concerns of persistent viruses leading to disease re-emergence with Ebola virus case study.

84. Mate SE, Kugelman JR, Nyenswah TG et al. Molecular evidence of sexual transmission of Ebola virus. N. Engl. J. Med. 373(25), 2448-2454 (2015).

85. Jacobs M, Rodger A, Bell DJ et al. Late Ebola virus relapse causing meningoencephalitis: a case report. Lancet 388(10043), 498-503 (2016).

86. Palma EL, Huang A. Cyclic production of vesicular stomatitis virus caused by defective interfering particles. J. Infect. Dis. 129(4), 402-410 (1974).

87. Frensing T, Heldt FS, Pflugmacher A et al. Continuous influenza virus production in cell culture shows a periodic accumulation of defective interfering particles. PLoS ONE 8(9), e72288 (2013).

88. Cave DR, Hendrickson FM, Huang AS. Defective interfering virus particles modulate virulence. J. Virol. 55(2), 366-373 (1985).

89. Stauffer Thompson KA, Rempala GA, Yin J. Multiple-hit inhibition of infection by defective interfering particles. J. Gen. Virol. 90(Pt 4), 888-899 (2009).

90. Thompson KA, Yin J. Population dynamics of an RNA virus and its defective interfering particles in passage cultures. Virol. J. 7, 257 (2010).

91. Moscona A. Defective interfering particles of human parainfluenza virus type 3 are associated with persistent infection in cell culture. Virology 183(2), 821-824 (1991).

92. Xu J, Sun Y, Li Y et al. Replication defective viral genomes exploit a cellular pro-survival mechanism to establish paramyxovirus persistence. Nat. Commun. 8(1), 799 (2017).

-• Description of a molecular mechanism by which DVGs lead to establishment of persistence.

93. Brenner D, Blaser H, Mak TW. Regulation of tumour necrosis factor signalling: live or let die. Nat. Rev. Immunol. 15(6), 362-374 (2015).

94. Moscona A, Peluso RW. Persistent infection with human parainfluenza virus 3 in CV-1 cells: analysis of the role of defective interfering particles. Virology 194(1), 399-402 (1993). 
